\title{
Soft X-Ray Second Harmonic Generation as an Interfacial Probe
}

R. K. Lam, ${ }^{1,2}$ S. L. Raj, ${ }^{1,2}$ T. A. Pascal, ${ }^{3}$ C. D. Pemmaraju, ${ }^{4}$ L. Foglia,${ }^{5}$ A. Simoncig, ${ }^{5}$ N. Fabris,${ }^{6,7}$ P. Miotti,${ }^{6,7}$ C. J. Hull, ${ }^{1,2}$ A. M. Rizzuto, ${ }^{1,2}$ J. W. Smith, ${ }^{1,2}$ R. Mincigrucci, ${ }^{5}$ C. Masciovecchio, ${ }^{5}$ A. Gessini, ${ }^{5}$ E. Allaria, ${ }^{5}$ G. De Ninno, ${ }^{5,8}$ B. Diviacco, ${ }^{5}$ E. Roussel, ${ }^{5}$ S. Spampinati ${ }^{5}$ G. Penco, ${ }^{5}$ S. Di Mitri, ${ }^{5}$ M. Trovò, ${ }^{5}$ M. Danailov, ${ }^{5}$ S. T. Christensen, ${ }^{9}$ D. Sokaras, ${ }^{10}$ T.-C. Weng, ${ }^{11}$ M. Coreno, ${ }^{5,12}$ L. Poletto, ${ }^{6}$ W. S. Drisdell, ${ }^{2}$ D. Prendergast,${ }^{3}$ L. Giannessi, ${ }^{5,13}$ E. Principi, ${ }^{5}$ D. Nordlund, ${ }^{10}$ R. J. Saykally, ${ }^{1,2, *}$ and C. P. Schwartz ${ }^{3,10, \dagger}$

${ }^{1}$ Department of Chemistry, University of California, Berkeley, California 94720, USA

${ }^{2}$ Chemical Sciences Division, Lawrence Berkeley National Laboratory, Berkeley, California 94720, USA

${ }^{3}$ The Molecular Foundry, Lawrence Berkeley National Laboratory, Berkeley, California 94720, USA

${ }^{4}$ Theory Institute for Materials and Energy Spectroscopies, SLAC National Accelerator Laboratory, Menlo Park, California 94025, USA

${ }^{5}$ Elettra-Sincrotrone Trieste S.C.p.A., Strada Statale 14—km 163.5, 34149 Trieste, Italy

${ }^{6}$ Institute of Photonics and Nanotechnologies, National Research Council of Italy, via Trasea 7, I-35131 Padova, Italy

${ }^{7}$ Department of Information Engineering, University of Padova, via Gradenigo 6/B, I-35131 Padova, Italy

${ }^{8}$ Laboratory of Quantum Optics, University of Nova Gorica, 5001 Nova Gorica, Slovenia

${ }^{9}$ National Renewable Energy Laboratory, Golden, Colorado 80401, USA

${ }^{10}$ SLAC National Accelerator Laboratory, Menlo Park, California 94025, USA

${ }^{11}$ Center for High Pressure Science \& Technology Advanced Research, Pudong, Shanghai 201203, China

${ }^{12}$ ISM-CNR, Elettra Laboratory, Basovizza, I-34149 Trieste, Italy

${ }^{13}$ ENEA, C.R. Frascati, Via E. Fermi 45, 00044 Frascati (Rome), Italy

(Received 11 October 2017; published 8 January 2018)

\begin{abstract}
Nonlinear optical processes at soft $\mathrm{x}$-ray wavelengths have remained largely unexplored due to the lack of available light sources with the requisite intensity and coherence. Here we report the observation of soft x-ray second harmonic generation near the carbon $K$ edge $(\sim 284 \mathrm{eV})$ in graphite thin films generated by high intensity, coherent soft x-ray pulses at the FERMI free electron laser. Our experimental results and accompanying first-principles theoretical analysis highlight the effect of resonant enhancement above the carbon $K$ edge and show the technique to be interfacially sensitive in a centrosymmetric sample with second harmonic intensity arising primarily from the first atomic layer at the open surface. This technique and the associated theoretical framework demonstrate the ability to selectively probe interfaces, including those that are buried, with elemental specificity, providing a new tool for a range of scientific problems.
\end{abstract}

DOI: 10.1103/PhysRevLett.120.023901

Nonlinear optics has recently been extended from visible and near UV wavelengths to new regimes with the development of $\mathrm{x}$-ray free electron lasers (XFELs) capable of delivering $\mathrm{X}$-ray pulses with high brightness, ultrashort pulse duration, and high coherence [1-7]. One fundamental nonlinear probe is second harmonic generation (SHG), a secondorder process which combines two photons of the same energy to generate a single photon with twice the energy [8]. At infrared, visible, and ultraviolet wavelengths, secondorder nonlinear spectroscopies have become important tools in surface science, as symmetry considerations within the dipole approximation constrain signal generation to regions lacking centrosymmetry, such as surfaces and interfaces [912]. In contrast, at hard X-ray wavelengths, second harmonic and sum frequency generation (SFG) have been observed in centrosymmetric materials with a nonuniform electron density and are essentially bulk probes $[1,2]$. As soft x-ray wavelengths fall in between the hard $\mathrm{x}$-ray and UV regimes, there has been uncertainty regarding the interface specificity of soft x-ray SHG. Here, by utilizing a recently constructed, highly coherent, soft $x$-ray free electron laser $[13,14]$, we report the first observation of soft $\mathrm{x}$-ray second harmonic generation near the carbon $K$ edge $(\sim 284 \mathrm{eV})$. Our experimental results and accompanying theoretical analysis indicate that soft x-ray SHG is an interface-specific probe with symmetry constraints similar to optical SHG or SFG, and is highly sensitive to resonance effects. This enables a powerful new approach for surface and interface analysis with broad applicability to many scientific fields, as it combines the elemental and chemical specificity of x-ray absorption spectroscopy with the rigorous interfacial specificity of second-order nonlinear spectroscopies, while maintaining a fully coherent signal. With several new coherent free electron lasers under development (SwissFEL, SXFEL, LCLS-II, FLASH2020) [15,16], this new technique offers exciting applications to a wide range of problems.

At optical wavelengths, second-order nonlinear spectroscopies have become indispensable techniques for surface 
analysis, being among the few techniques with genuine interface specificity. As such, SHG and SFG have been employed to study of a wide range of scientifically important systems (e.g., liquid-vapor interfaces, catalytic interfaces, etc.) [11]. These techniques exploit the enhancement of the second-order nonlinear response to a high intensity, coherent photon beam when either the intermediate or final state is resonant with a real state within the target material. The nonlinear response to a coherent input beam of frequency can be derived from an expansion of the polarization of the target medium

$$
\vec{P}=\vec{X}^{(1)} E+\vec{X}^{(2)} E E+\vec{X}^{(3)} E E E+\cdots,
$$

where $\vec{X}^{(n)}$ is the $n$ th-order susceptibility tensor. Within the dipole approximation, even orders of $\vec{X}^{(n)}$ necessarily vanish in centrosymmetric media. This causes SHG, SFG, difference frequency generation, and higher evenorder nonlinear signals to be inherently surface specific in centrosymmetric media, as this symmetry is broken only at interfaces. Assuming a broken-symmetry region with thickness much less than the wavelength, the intensity of the SHG signal from a given interface is given by

$$
I_{2 \omega} \propto\left|X_{\mathrm{eff}}^{(2)}\right|^{2} I_{\omega}^{2},
$$

where $X_{\mathrm{eff}}^{(2)}$ is the effective susceptibility of the interfacial region, and $I_{\omega}$ is the intensity of the fundamental beam. Note the quadratic dependence of the second harmonic $(\mathrm{SH})$ intensity $I_{2 \omega}$ on the input intensity $I_{\omega}$.

Until recently, the lack of light sources with sufficient intensity and coherence has limited the development of nonlinear x-ray spectroscopies. Prior to the development of free electron lasers (FELs), only parametric downconversion had been observed [17]. While the advent of
XFELs has recently enabled second- and third-order nonlinear spectroscopies at hard $\mathrm{x}$-ray energies, including SHG [2], SFG [1], two-photon absorption [4], and inelastic Compton scattering [18], current hard x-ray FELs lack the longitudinal and temporal coherence necessary for efficiently satisfying the phase-matching conditions required for nonlinear spectroscopies, thus, making the exploitation of some of these techniques difficult $[19,20]$. Furthermore, the shorter hard $\mathrm{x}$-ray wavelengths $(\lambda<0.2 \mathrm{~nm})$ induce second harmonic and sum frequency generation even within centrosymmetric media, as the observed response depends on material inhomogeneity on the length scale of the x-ray wavelength, similar to how SHG is seen in a plasma, and effectively making this method a bulk probe [21].

Here we report the observation of soft x-ray SHG generated by high intensity, coherent FEL pulses at FERMI, a highly coherent soft $\mathrm{x}$-ray FEL based on highgain harmonic generation of a longer wavelength seed laser $[13,14]$. The experiments were conducted at the EIS-TIMEX end station [22,23]. An experimental schematic is shown in Fig. 1(a): a soft x-ray FEL pulse (estimated pulse duration $\sim 25 \mathrm{fs}$, pulse energy $\sim 1-5 \mu \mathrm{J}$, spot size $\sim 350 \mu \mathrm{m}^{2}$ ) is passed through a $2 \mathrm{~mm}$ iris to attenuate the off-axis second harmonic contamination from the FEL source and focused onto a graphite sample at normal incidence. The transmitted beam and the collinear $\mathrm{SH}$ signal are passed through a $600 \mathrm{~nm}$ aluminum filter to attenuate the fundamental FEL pulse into a spectrograph [24] to separate the $\mathrm{SH}$ signal from the fundamental, and onto a CCD detector [Fig. 1(b)]. The sample was raster scanned so that pristine graphite was probed with each laser pulse. Measurements were made using three discrete wavelengths chosen to have incoming photon energies lying below $(\lambda=4.76 \mathrm{~nm}, \quad \hbar \omega=$ $260.5 \mathrm{eV})$ on $(\lambda=4.36 \mathrm{~nm}, \hbar \omega=284.18 \mathrm{eV})$, and above $(\lambda=4.02 \mathrm{~nm}, \quad \hbar \omega=307.86 \mathrm{eV})$ the carbon $K$-edge
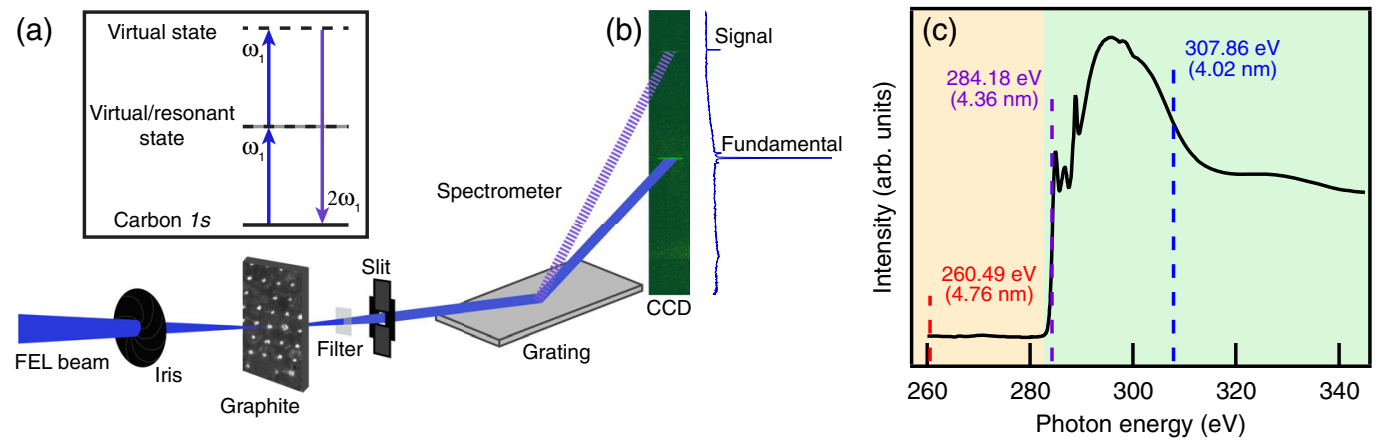

FIG. 1. Experimental design. (a) X-ray pulses are passed through a $2 \mathrm{~mm}$ iris, then focused by an ellipsoidal mirror (not shown) onto the graphite sample (spot size $\sim 350 \mu \mathrm{m}^{2}$ ) at normal incidence. The transmitted beam and the collinear second harmonic signal (double the fundamental photon energy) are passed through a $600 \mathrm{~nm}$ aluminum filter and into a spectrometer, spatially separating the second harmonic signal from the fundamental. A schematic energy level diagram of the SHG process is shown in the inset. (b) The CCD image and projection of the transmitted FEL beam and the SHG signal for a single FEL pulse. (c) Linear, total electron yield x-ray absorption spectrum of a $500 \mathrm{~nm}$ graphite sample. X-ray SHG measurements were made at the three discrete photon energies $\left(\omega_{1}\right)$ shown in the dashed lines. The nonresonant (below the $\mathrm{C} K$ edge) and resonant (above the $\mathrm{C} K$ edge) regions are shaded in orange and green, respectively. 
absorption edge of graphite [see Fig. 1(c)] to demonstrate the effects of resonant enhancement. In this case, the FEL energy is resonant with the intermediate state and generates a nonresonant signal enabling transmission experiments. SHG spectra can also be measured at half the photon energy used here, where the final state is resonant, although this would hinder transmission studies. Measurements were made both with and without the sample present to permit subtraction of any remaining second harmonic contamination from the FEL beam. See the Supplemental Material for additional details on the experimental design and analysis procedures [25].

The measured dependence of the second harmonic signal on the pulse energy for multiple thicknesses of graphite $(100-720 \mathrm{~nm})$ corresponding to $260.49,284.18$, and $307.86 \mathrm{eV}$ is shown in Figs. 2(a), 2(b), and 2(c), respectively. The pulse energy was calculated from the absolute number of $\mathrm{x}$-ray photons incident on the sample, which was directly measured by the spectrometer with the sample removed. A quadratic fit to the $\mathrm{SH}$ response with respect to input intensity at a given photon energy is represented by a dashed line. At each photon energy, the power dependence of the SH signal is largely invariant with the sample thickness, an observation that is consistent with surface sensitivity in a

(a)

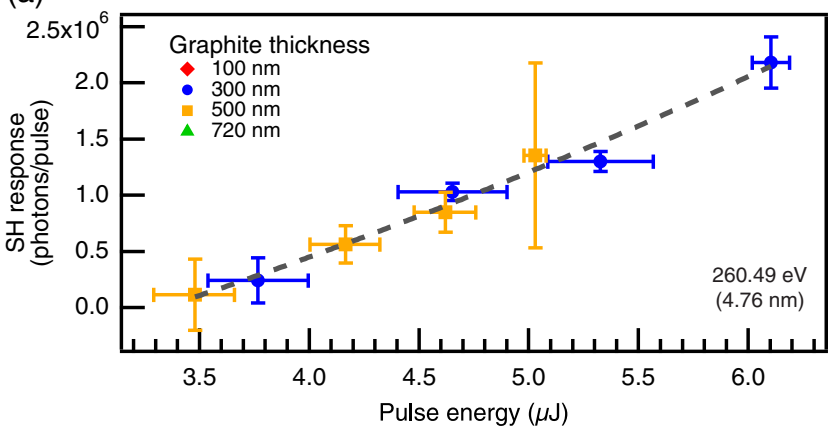

(b)

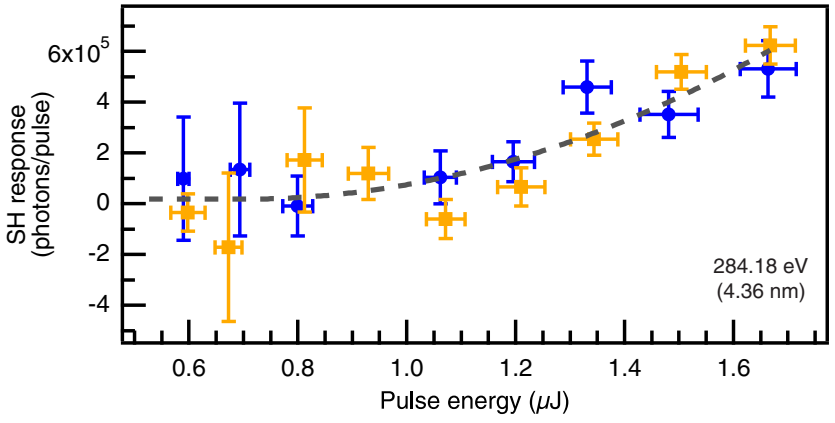

centrosymmetric sample. Notably, even for thin samples with a large transmission (>70\%), a thickness-dependent increase in the SH signal is not observed, implying that the signal is not bulk-volume dependent. This invariance with sample thickness is also observed at resonant photon energies. The bulk second harmonic signal in graphite due to electric quadrupole contributions is weak.

At the resonant photon energies, a significant enhancement of the SH signal was observed, permitting its detection with significantly lower pulse energies. The tunable nature of FELs facilitates resonance and double resonance exploitation. The relative $\left|X_{\text {eff }}^{(2)}\right|^{2}$ response of graphite at different $\mathrm{x}$-ray wavelengths is proportional to the slope of the linearized power dependence curve ( $\mathrm{SH}$ response vs [input power] ${ }^{2}$ ) shown in Fig. 2(d). A significant enhancement in the $X_{\text {eff }}^{(2)}$ response is observed when exciting with $\mathrm{x}$-ray energies at or above the graphite absorption onset $(>284 \mathrm{eV})$. In this case, the intermediate state is resonant with discrete transitions to unoccupied electronic states [Fig. 1(c)], and the data are consistent with the expected resonant enhancement of the SH signal when either the intermediate or final state is real. When off resonance at $260.49 \mathrm{eV}$, a threshold pulse energy of over

(c)

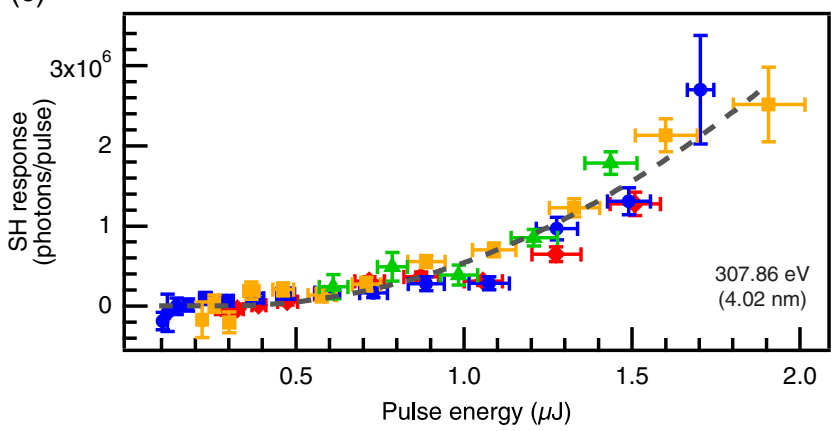

(d)

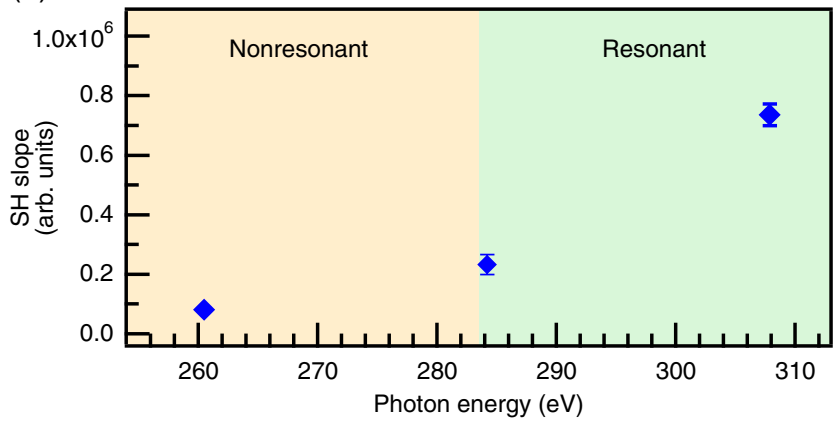

FIG. 2. Pulse energy dependence of soft x-ray SHG. The second harmonic response at 260.49 (a), 284.18 (b), and $307.86 \mathrm{eV}$ (c) of graphite thin films $(100 \mathrm{~nm}$, red diamond; $300 \mathrm{~nm}$, blue circle; $500 \mathrm{~nm}$, orange square; $720 \mathrm{~nm}$, green triangle). The dashed lines represent quadratic fits to all the points at a given photon energy constrained to show no detectable second harmonic signal generated below a threshold pulse energy. The vertical and horizontal error bars represent the standard error of the SH response and the standard deviation of the pulse energies in each bin, respectively. (d) The slope of the linearized pulse energy dependence curve for each photon energy. The relative $\left|X_{\text {eff }}^{(2)}\right|^{2}$ response is proportional to the slope of the linearized pulse energy dependence curve (SH response vs $[\text { input power }]^{2}$ ). Error bars represent the standard deviation from the linear regression analysis. The nonresonant and resonant regions are shaded in orange and green, respectively. 
$3 \mu \mathrm{J}$ per pulse is required for a sufficient SH signal-to-noise ratio as determined based on the minimum of the quadratic fits shown in Fig. 2. On resonance, the pulse energy necessary is significantly lower, viz., 0.7 and $0.3 \mu \mathrm{J}$ at 284.18 and $307.86 \mathrm{eV}$, respectively. This resonance enhancement means that the technique can be used to generate a surface-specific soft x-ray spectrum with sensitivity to chemical composition. The pump intensities used here correspond to peak fields of $10^{12} \mathrm{~W} / \mathrm{cm}^{2}$, which is 4 orders of magnitude less than what was required for hard $\mathrm{x}$ ray SHG $\left(10^{16} \mathrm{~W} / \mathrm{cm}^{2}\right)$ [2]. Note that at these power densities, the efficiency of the SHG process is at least an order of magnitude lower than the linear absorption for a single atomic layer [36].

While the exact damage threshold will vary based on the sample, we expect that it will be possible to perform soft $\mathrm{x}$-ray SFG below the damage threshold for a broad variety of materials by leveraging the lower $\mathrm{x}$-ray fields required by combining the soft $\mathrm{X}$-ray pulse with an optical laser pulse.
When hard x-ray SFG was performed by using an optical laser pulse combined with an x-ray laser, the pump intensities were $\sim 10^{10} \mathrm{~W} / \mathrm{cm}^{2}$ for the optical laser and $10^{11} \mathrm{~W} / \mathrm{cm}^{2}$ for the $\mathrm{x}$-ray laser [1]. Additionally, as a soft $\mathrm{x}$-ray SHG requires significantly smaller fields than does a hard x-ray SHG, such damage-free SHG measurements may be achievable by exploiting higher repetition rate FELs (LCLS-II, FLASH2020) and more efficient detection (better spectrometer, spatial separation, etc.).

In order to verify our experimental observation of a significant SHG response in the soft $\mathrm{x}$-ray regime of graphite, we calculated the linear and second-order response from first principles using perturbation theory within density functional theory $[37,38]$. This approach is broadly applicable for calculating electronic SHG and SFG spectra from the optical regime through the x-ray energies and is detailed in the Supplemental Material [25]. Figures 3(a) and 3(b) present the linear and SH response, respectively, of an eight-layer slab of $A B$ stacked hexagonal

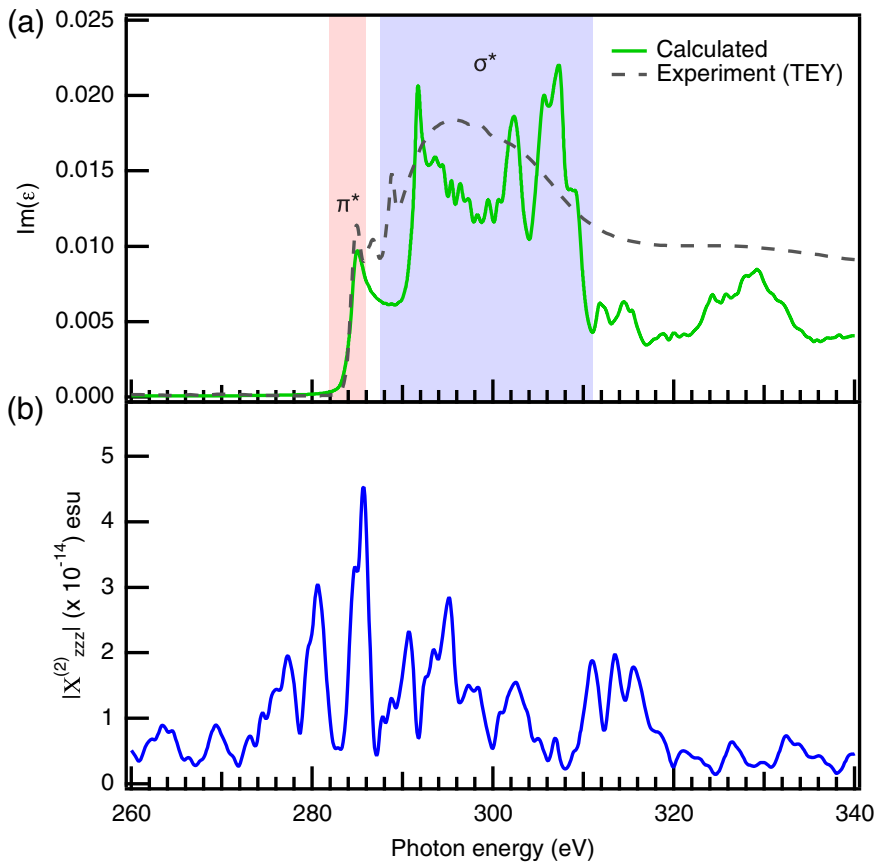

FIG. 3. Second harmonic susceptibility from first-principles theory. (a) Imaginary part (green solid) of the linear dielectric function corresponding to the calculated linear spectrum at the $\mathrm{C} K$-edge NEXAFS region calculated with a density-functional-theory-based supercell approach for an eight-layer slab of graphite compared with the experimental linear spectrum (gray, dashed). The $\pi^{*}$ and $\sigma^{*}$ transitions are labeled in red and blue, respectively. The experimental peak between the marked $\pi^{*}$ and $\sigma^{*}$ regions corresponds to oxidized graphite. Note that a more realistic comparison between theory and experiment can be obtained by sampling a finite temperature molecular dynamics simulation as inhomogeneity in thermalized samples is a significant contributor to spectral broadening.

(b) Calculated C $1 s$ core level contribution to the magnitude $\left|X_{z z z}^{(2)}\right|$ of the $z z z$ component of the second-order nonlinear susceptibility tensor $X^{(2)}(2 \omega, \omega, \omega)$ relevant to SHG near the C $K$ edge of a graphite surface. C $1 s$ core levels from the top four surface layers of an eight-layer slab of graphite [shown in (d)] are considered in the simulation. The $z$ axis is perpendicular to the surface of the slab. (c) Convergence of the calculated $\left|X_{z z z}^{(2)}\right|$ as a function of the number of surface layers whose $\mathrm{C} 1 s$ core states are included in the simulation. The inset shows the percentage contribution to $\left|X_{z z z}^{(2)}\right|$ from each of the top four individual layers obtained by integrating $\left|X_{z z z}^{(2)}\right|$ over a $260-340 \mathrm{eV}$ energy window plotted as a function of the layer index. Contribution to the overall response is seen to decay exponentially moving into the bulk of the slab with the top layer accounting for $\sim 63 \%$ of the signal. (d) Periodic supercell of the eightlayer graphite slab employed in the simulations. 
graphite. A better comparison to experiments can be obtained by sampling a finite temperature molecular dynamics trajectory, as has been demonstrated for linear absorption [39]. Nevertheless, the linear absorption spectrum calculated here within the independent particle approximation captures salient resonant features, such as $\pi^{*}$ and $\sigma^{*}$ features at 284.9 and $291.65 \mathrm{eV}$, compared to the respective experimental values of 284.9 and $288.8 \mathrm{eV}$. Because of symmetry considerations, only the $X_{z z z}$ component of the $X^{(2)}$ susceptibility tensor is nonvanishing. Small changes in the energy calibration and temperature effects in the theory and the imperfect materials used in the experiments can engender large differences in signal intensity, particularly near the absorption edge, likely accounting for the observed differences between experiment and theory. The main features in the SH spectrum correspond to excitations from a convolution of the ground state and $\pi^{*}$ and $\sigma^{*}$ intermediate states in the conduction band. As such, a direct comparison between the SH spectrum and the linear spectrum is infeasible.

The signal originating from each layer of the graphite slab was isolated in order to determine the exact degree of surface specificity. As shown in the inset of Fig. 3(c), the contribution to the overall response decays exponentially as a function of the layer index and is essentially limited to the first three monolayers, or $\sim 0.5 \mathrm{~nm}$, with $\sim 63 \%$ of the signal originating from the topmost layer, $\sim 25 \%$ from the second, and $\sim 9 \%$ from the third. This result is consistent with expectations from optical sum frequency generation, wherein $90 \%$ of the signal originates from the first two molecular layers [40].

In conclusion, soft $\mathrm{x}$-ray second harmonic generation is demonstrated to be a powerful elemental- and chemicalspecific spectroscopy that is highly symmetry sensitive. More specifically, the interfacial nonlinearity is large enough to prevail over the plasma nonlinearities that are dominant at hard $\mathrm{x}$-ray wavelengths. This new technique will permit a new class of interface analysis experiments with significantly higher interfacial specificity than existing soft x-ray methods, effectively allowing for x-ray absorption spectroscopy of the first few molecular layers of an exposed bulk sample or either side of a buried interface. For example, buried interfaces in layered samples, wherein an individual layer contains an element not present in the others, could be probed directly without concomitant contamination from the exposed interfaces. Additionally, as the pulses remain fully coherent, it should be possible to combine this technique with lensless coherent imaging techniques with a resolution not limited by aberration [41]. Such a technique would require either an energy-sensitive nondispersive detector or the generation of a signal that is spatially separated from the input pulses, as has been achieved previously with noncollinear input laser pulses, such as in optical SFG [9-12]. If radiation damage concerns can be mitigated, the spectral sensitivity demonstrated here would enable experiments which can both spectroscopically and spatially resolve nanoparticles. Such measurements, when combined with the first-principles electronic structure framework developed here, will have broad applicability to a variety of systems of current interest.

Soft x-ray SHG measurements were conducted at the EIS-TIMEX beam line at FERMI. R. K. L., A. M. R., J. W. S., and R. J.S. were supported by the Office of Science, Office of Basic Energy Sciences, Division of Chemical Sciences, Geosciences, and Biosciences of the U.S. Department of Energy at the Lawrence Berkeley National Laboratory under Contract No. DE-AC0205CH11231 (LBNL). C. J. H. and S. L. R. were supported by the U.S. Army Research Laboratory (ARL) and the U.S. Army Research Office (ARO) under Contracts/Grants No. W911NF-13-1-0483 and No. W911NF-17-1-0163. S. L. R. received a National Science Foundation Graduate Research Fellowship under Grant No. DGE 1106400. Any opinions, findings, and conclusions or recommendations expressed in this material are those of the author(s) and do not necessarily reflect the views of the National Science Foundation. Simulations were performed as part of a user project with T. A. P., C. D. P., and D. P. at The Molecular Foundry (TMF), LBNL. Theoretical simulations of nonlinear susceptibility by C. D. P. were carried out within TIMES at SLAC National Accelerator Laboratory supported by the U.S. Department of Energy, Office of Basic Energy Sciences, Division of Materials Sciences and Engineering, under Contract No. DE-AC0276SF00515. The calculations were performed using computational resources at the National Energy Research Scientific Computing Center and at TMF, both Department of Energy Office of Science User Facilities at LBNL supported by the Office of Science of the U.S. Department of Energy under the contract listed above. N. F., P. M., M. C., and L. P. were supported by the project Single-Shot X-Ray Emission-Spectroscopy experiments funded by the Italian Ministry for Education and Research as an in-kind project for the EuroFEL consortium. W. S. D. was supported by the Joint Center for Artificial Photosynthesis, a Department of Energy Energy Innovation Hub supported through the Office of Science of the U.S. Department of Energy under Award No. DE-SC0004993. S. T. C. was supported by U.S. Department of Energy under Contract No. DE-AC36-08-GO28308 with the National Renewable Energy Laboratory. D. S., T.-C. W., D. N., S. T. C., and C.P.S. were in part supported by the U.S. Department of Energy, Office of Energy Efficiency \& Renewable Energy, Solar Energy Technology Office BRIDGE program. D. S., T.-C. W., D. N., and C. P. S. were also supported by the SLAC National Accelerator Laboratory. Electron yield x-ray absorption measurements were performed at Stanford Synchrotron Radiation Lightsource. Use of the Stanford Synchrotron Radiation 
Lightsource, SLAC National Accelerator Laboratory is supported by the U.S. Department of Energy, Office of Science, Office of Basic Energy Sciences under Contract No. DE-AC02-76SF00515. Material support was provided by the Office of Science, Office of Basic Energy Sciences, Division of Chemical Sciences, Geosciences, and Biosciences of the U.S. Department of Energy at LBNL under the contract listed above. Travel support was provided by ARL and ARO under the Contracts/Grants listed above.

*saykally@ berkeley.edu †cpschwartz@lbl.gov

[1] T. E. Glover, D. M. Fritz, M. Cammarata, T. K. Allison, S. Coh, J. M. Feldkamp, H. Lemke, D. Zhu, Y. Feng, R. N. Coffee, M. Fuchs, S. Ghimire, J. Chen, S. Shwartz, D. A. Reis, S. E. Harris, and J. B. Hastings, Nature (London) 488, 603 (2012).

[2] S. Shwartz, M. Fuchs, J. B. Hastings, Y. Inubushi, T. Ishikawa, T. Katayama, D. A. Reis, T. Sato, K. Tono, M. Yabashi, S. Yudovich, and S. E. Harris, Phys. Rev. Lett. 112, 163901 (2014).

[3] N. Rohringer, D. Ryan, R. A. London, M. Purvis, F. Albert, J. Dunn, J. D. Bozek, C. Bostedt, A. Graf, R. Hill, S. P. HauRiege, and J. J. Rocca, Nature (London) 481, 488 (2012).

[4] K. Tamasaku, E. Shigemasa, Y. Inubushi, T. Katayama, K. Sawada, H. Yumoto, H. Ohashi, H. Mimura, M. Yabashi, K. Yamauchi, and T. Ishikawa, Nat. Photonics 8, 313 (2014).

[5] F. Bencivenga, F. Capotondi, R. Mincigrucci, R. Cucini, M. Manfredda, E. Pedersoli, E. Principi, A. Simoncig, and C. Masciovecchio, Phys. Scr. T169, 014003 (2016).

[6] F. Bencivenga, R. Cucini, F. Capotondi, A. Battistoni, R. Mincigrucci, E. Giangrisostomi, A. Gessini, M. Manfredda, I. P. Nikolov, E. Pedersoli, E. Principi, C. Svetina, P. Parisse, F. Casolari, M. B. Danailov, M. Kiskinova, and C. Masciovecchio, Nature (London) 520, 205 (2015).

[7] J. Szlachetko, J. Hoszowska, J.-C. Dousse, M. Nachtegaal, W. Błachucki, Y. Kayser, J. Sà, M. Messerschmidt, S. Boutet, G. J. Williams, C. David, G. Smolentsev, J. A. van Bokhoven, B. D. Patterson, T. J. Penfold, G. Knopp, M. Pajek, R. Abela, and C. J. Milne, Sci. Rep. 6, 33292 (2016).

[8] P. A. Franken, A. E. Hill, C. W. Peters, and G. Weinreich, Phys. Rev. Lett. 7, 118 (1961).

[9] K. B. Eisenthal, Annu. Rev. Phys. Chem. 43, 627 (1992).

[10] A. M. Rizzuto, S. Irgen-Gioro, A. Eftekhari-Bafrooei, and R. J. Saykally, J. Phys. Chem. Lett. 7, 3882 (2016).

[11] Y.-R. Shen, Fundamentals of Sum-Frequency Spectroscopy (Cambridge University Press, Cambridge, England, 2016).

[12] H.-F. Wang, W. Gan, R. Lu, Y. Rao, and B.-H. Wu, Int. Rev. Phys. Chem. 24, 191 (2005).

[13] E. Allaria et al., Nat. Photonics 6, 699 (2012).

[14] E. Allaria et al., Nat. Photonics 7, 913 (2013).

[15] J. Galayda, Linac14, Geneva, Switzerland (2014), http:// accelconf.web.cern.ch/AccelConf/linac2014/.
[16] M. Song, C. Feng, D. Huang, H. Deng, B. Liu, and D. Wang, Nucl. Sci. Tech. 28, 90 (2017).

[17] S. Shwartz, R. N. Coffee, J. M. Feldkamp, Y. Feng, J. B. Hastings, G. Y. Yin, and S. E. Harris, Phys. Rev. Lett. 109, 013602 (2012).

[18] M. Fuchs et al., Nat. Phys. 11, 964 (2015).

[19] I. A. Vartanyants et al., Phys. Rev. Lett. 107, 144801 (2011).

[20] L.-H. Yu et al., Science 289, 932 (2000).

[21] A. Nazarkin, S. Podorov, I. Uschmann, E. Förster, and R. Sauerbrey, Phys. Rev. A 67, 041804 (2003).

[22] A. Simoncig, R. Mincigrucci, E. Principi, F. Bencivenga, A. Calvi, L. Foglia, G. Kurdi, L. Raimondi, M. Manfredda, N. Mahne, R. Gobessi, S. Gerusina, C. Fava, M. Zangrando, A. Matruglio, S. Dal Zilio, V. Masciotti, and C. Masciovecchio, Proc. SPIE Int. Soc. Opt. Eng. 10243, 102430L (2017).

[23] C. Masciovecchio et al., J. Synchrotron Radiat. 22, 553 (2015).

[24] L. Poletto, F. Frassetto, P. Miotti, A. Di Cicco, P. Finetti, C. Grazioli, F. Iesari, A. Kivimäki, S. Stagira, and M. Coreno, Rev. Sci. Instrum. 85, 103112 (2014).

[25] See Supplemental Material at http://link.aps.org/ supplemental/10.1103/PhysRevLett.120.023901 for additional details on the experimental design, data analysis procedure, and theoretical methods, which includes Refs. [26-35].

[26] P. Finetti et al., Phys. Rev. X 7, 021043 (2017).

[27] M. Zangrando, D. Cocco, C. Fava, S. Gerusina, R. Gobessi, N. Mahne, E. Mazzucco, L. Raimondi, L. Rumiz, and C. Svetina, J. Synchrotron Radiat. 22, 565 (2015).

[28] P. Hohenberg and W. Kohn, Phys. Rev. 136, B864 (1964).

[29] W. Kohn and L. J. Sham, Phys. Rev. 140, A1133 (1965).

[30] P. Trucano and R. Chen, Nature (London) 258, 136 (1975).

[31] N. Ooi, A. Rairkar, and J. B. Adams, Carbon 44, 231 (2006).

[32] J. P. Perdew and A. Zunger, Phys. Rev. B 23, 5048 (1981).

[33] S. Klinkusch, P. Saalfrank, and T. Klamroth, J. Chem. Phys. 131, 114304 (2009).

[34] A. P. Sorini, J. J. Kas, J. J. Rehr, M. P. Prange, and Z. H. Levine, Phys. Rev. B 74, 165111 (2006).

[35] C. Kunz, B. Cowie, W. Drube, T.-L. Lee, S. Thiess, C. Wild, and J. Zegenhagen, J. Electron Spectrosc. Relat. Phenom. 173, 29 (2009).

[36] B. L. Henke, E. M. Gullikson, and J. C. Davis, At. Data Nucl. Data Tables 54, 181 (1993).

[37] S. Sharma and C. Ambrosch-Draxl, Phys. Scr. T109, 128 (2004).

[38] A. Gulans, S. Kontur, C. Meisenbichler, D. Nabok, P. Pavone, S. Rigamonti, S. Sagmeister, U. Werner, and C. Draxl, J. Phys. Condens. Matter 26, 363202 (2014).

[39] J.-J. Velasco-Velez, C. H. Wu, T. A. Pascal, L. F. Wan, J. Guo, D. Prendergast, and M. Salmeron, Science 346, 831 (2014).

[40] A. Morita and J. T. Hynes, Chem. Phys. 258, 371 (2000).

[41] J. Miao, P. Ercius, and S. J. L. Billinge, Science 353, aaf2157 (2016). 